\title{
High pressure rate constants for unimolecular dissociation/free radical recombination: Determination of the quantum correction via quantum Monte Carlo path integration
}

\author{
Stephen J. Klippenstein and R. A. Marcus \\ Arthur Amos Noyes Laboratory of Chemical Physics, California Institute of Technology, ${ }^{\text {a) Pasadena, }}$ \\ California 91125
}

(Received 20 March 1987; accepted 2 June 1987)

\begin{abstract}
The determination of a quantum correction factor for the transitional modes of a unimolecular dissociation/free radical recombination reaction having a transition state of varying looseness is described. The quantum correction factor for the high pressure canonical rate constant is calculated via Monte Carlo path integral evaluation of partition function ratios, and is applied to the recombination reaction $2 \mathrm{CH}_{3} \rightarrow \mathrm{C}_{2} \mathrm{H}_{6}$.
\end{abstract}

\section{INTRODUCTION}

In RRKM theory the (microcanonical) unimolecular rate constant $k_{E J}$ is given by ${ }^{1-3}$

$$
k_{E J}=\frac{N_{E J}^{\dagger}}{h \rho_{E J}},
$$

where $N_{E J}^{+}$is the number of states of the transition state with energy equal to or less than $E$, and with a given total angular momentum $J ; \rho_{E J}$ is the density of states for the reactants at the given $E$ and $J$. In the case of a unimolecular dissociation of the parent molecule into two particles, the coordinates which contribute to $N_{E}^{+}$are largely of two types: (i) vibrational modes, usually of high frequency, whose characteristic motion does not change appreciably from the parent molecule to the transition state, and (ii) the remaining modes whose characteristic motion does change. The latter consist, typically, of coupled degrees of freedom which are fragment bending plus overall rotational motions, and which subsequently become hindered relative rotations plus overall rotational motion in the transition state. In the fragment molecules these modes are typically the free rotations and relative translation of the two fragments. The fragment-fragment stretching in the parent molecule typically becomes the reaction coordinate itself.

Wardlaw and Marcus ${ }^{4-7}$ separated the modes into these "conserved" and "transitional" modes, respectively. In this separation the conserved modes were treated quantum mechanically and the transitional modes were treated classically. Their result for the number of states of the transition state for a given energy and total angular momentum $J$ was, thereby, given as the convolution

$$
N_{E J}^{\dagger}=\int_{0}^{E} N_{V}(E-\epsilon) \Omega_{J}(\epsilon) d \epsilon,
$$

where $N_{V}(E-\epsilon)$ is the number of states in the conserved modes with energy less than or equal to $E-\epsilon$, and $\Omega_{J}(\epsilon)$ is the number of states in the transitional modes with total angular momentum equal to $J$, and with energy in the range $\epsilon$ to $\epsilon+d \epsilon . N_{V}(E-\epsilon)$ was calculated via a direct quantum

\footnotetext{
a) Contribution No. 7565
}

count, while $\Omega_{J}(\epsilon)$ was obtained by transformation to action angle coordinates followed by "crude" Monte Carlo evaluation of the phase space integral. Purely classical Monte Carlo phase space volume calculations are described in, e.g., Refs. 8-17.

Many other approaches to the treatment of the transitional modes have been given previously. (See, e.g., Refs. 18-26.) However, the only treatment which has included all of the couplings within the transitional modes, while maintaining a quantum treatment for the conserved modes, appears to be that of Wardlaw and Marcus. ${ }^{4-7}$ In a recent article, Pacey ${ }^{27}$ has discussed and applied many of the approaches to the two-dimensional hindered rotor motion on a model sinusoidal potential. Comparison of the results obtained for the partition function from the various approaches with that determined from the exact quantum partition function for the same model potential shows the differences between the various approaches can be significant. In another recent study on the bending/rotation problem, Hase et $a l^{28}$ have compared rate constants for the $\mathrm{H}+\mathrm{CH}_{3} \rightarrow \mathrm{CH}_{4}$ recombination reaction with the transitional modes treated as either classical hindered rotors or harmonic oscillators (quantum or classical). They find that the partition functions and rate constants can vary by about $10 \%$ depending on how these modes are treated.

As noted in the studies of Wardlaw and Marcus, the question arises, what are the quantum corrections for the contribution of the transitional modes to the overall rate. When the transition state is nearly "loose" such a correction is expected to be minor. One direct approach to calculating the correction would be to determine the number of quantum states for the transitional modes as a function of energy and total angular momentum, and convolute that result with the quantum result for the "conserved" modes. This approach is currently under study. In the present manuscript, however, rather than looking at individual $k_{E J}$ 's, we have chosen to consider first the cumulative effect of the transitional modes by determining their quantum mechanical contribution to the high pressure canonical rate constant $k_{\infty}(T)$ defined below. The position of the transition state is determined variationally, ${ }^{29-31}$ namely, as that value (the "transition value") of the reaction coordinate for which the calculated $k_{\infty}(T)$ is a minimum. 
In the present study we note that $k_{\infty}(T)$ is given by a ratio of partition functions and so the quantum mechanical corrections, aside from quantum corrections due to tunneling along the reaction coordinate, may be determined by considering the Monte Carlo path integral evaluation of partition function ratios. The correction factor can thus be obtained for any potential energy surface and is for the canonical rate constant. In Sec. II, for comparison, the canonical rate constant is determined using quantum partition functions for the conserved modes and classical partition functions for the transitional modes. The determination of the quantum correction for the transitional modes is presented in Sec. III. In Sec. IV results for the methyl radical recombination reaction: $2 \mathrm{CH}_{3} \rightarrow \mathrm{C}_{2} \mathrm{H}_{6}$ are presented and discussed. The correction proves to be relatively small for the temperatures investigated.

\section{DETERMINATION OF $\boldsymbol{k}_{\infty}$ ( $\boldsymbol{\eta}$ ) USING CLASSICAL CANONICAL TST FOR THE TRANSITIONAL MODES}

The canonical rate constant $k_{\infty}(T)$ is given in terms of $N_{E J}^{\dagger} \mathrm{by}^{32}$

$k_{\infty}(T)=\frac{g_{e}}{h Q^{r}(T)} \int_{0}^{\infty} d E \sum_{J=0}^{\infty}(2 J+1) N_{E J}^{\dagger} \exp (-\beta E)$,

where $Q^{r}(T)$ is the partition function for the reactants (in the center-of-mass system and excluding electronic degrees of freedom ), $g_{e}$ is the ratio of the electronic partition function for the transition state to that for the reactants, and $\beta=1 / k_{B} T$. In a comparative study Wardlaw and Marcus have evaluated $k_{\infty}(T)$ at several levels. ${ }^{6}$ In the first method they determined the location of the transition state variationally for each $E$ and $J$ and then evaluated the sum and integral in Eq. (2.1) numerically. In a second calculation, which represents an approximation to the first, they determined the transition state location for each $E$ by minimizing a $J$-sampled integrand, and in a third calculation, which represents still a further approximation, they found the transition state location of an ensemble which sampled over $E$ and $J$ at a given $T$. They noted that the three $k_{\infty}(T)$ 's are related by $k_{\infty}^{\mathrm{I}}(T) \leqslant k_{\infty}^{\mathrm{II}}(T) \leqslant k_{\infty}^{\mathrm{III}}(T), k_{\infty}^{\mathrm{I}}(T)$ being the correct one. This last calculation, $k_{\infty}^{\mathrm{III}}(T)$, is equivalent ${ }^{1,33}$ to determining $k_{\infty}(T)$ variationally from the expression

$$
k_{\infty}(T)=g_{e} \frac{k_{B} T}{h} \frac{Q^{+}}{Q^{r}},
$$

where $Q^{\dagger}$ is the partition function for the transition state. The $Q^{\dagger}$ and $Q^{r}$ in Eq. (2.2) are calculated relative to the same energy zero. Separating the modes into the conserved modes $(c)$ and the transitional modes $(t)$ implies that

$$
k_{\infty}(T)=g_{e} \frac{k_{B} T}{h} \frac{Q_{c}^{\dagger}}{Q_{c}^{r}} \frac{Q_{t}^{\dagger}}{Q_{t}^{r}},
$$

where $Q_{c}^{\dagger}$ and $Q_{c}^{r}$ are the partition functions for the conserved modes at the transition state and at the reactant configuration, respectively. They can be evaluated using quantum oscillator partition functions. $Q_{t}^{\dagger}$ and $Q_{t}^{r}$ are the partition functions for the transitional modes at the transition state and at the reactant configuration, respectively.

For a free radical recombination reaction, the classical partition function for the transitional modes of the reactants $Q_{t}^{r}$ separates into the translational partition function for a particle of mass $\mu_{12}$, where $\mu_{12}$ is the reduced mass of the two fragments, and the product of the classical free-rotor partition functions for the two reactant fragments, $\Pi_{i=1}^{2} \sigma_{i}^{-1}\left[\pi\left(2 k_{B} T / \hbar^{2}\right)^{3} I_{A_{i}} I_{B_{i}} I_{C_{i}}\right]^{1 / 2}$, where $I_{A_{i}}, I_{B_{i}}$, and $I_{C_{i}}$ are the moments of inertia of reactant fragment $i$, and $\sigma_{i}$ is the symmetry number for reactant fragment $i$. The classical partition function for the transitional modes of the transition state is given by

$$
\begin{aligned}
Q_{t}^{\dagger}= & \frac{1}{\sigma^{\dagger}} \int d p_{\theta_{12}} d p_{\phi_{12}} d \theta_{12} d \phi_{12} \\
& \times\left(\prod_{i=1}^{2} d p_{\theta_{i}} d p_{\phi_{i}} d p_{\psi_{i}} d \theta_{i} d \phi_{i} d \psi_{i}\right) \exp \left(-\beta H_{t}\right),
\end{aligned}
$$

where $\sigma^{\dagger}$ is the symmetry factor for the transition state, $\theta_{i}, \phi_{i}$, and $\psi_{i}$ are the Euler angles ${ }^{34}$ for the absolute orientation in space of fragment $i$, and $\theta_{12}$ and $\phi_{12}$ describe the absolute orientation in space of the line of centers. $H_{t}$ is the classical Hamiltonian for the transitional degrees of freedom and is given by

$$
\begin{aligned}
H_{t}= & \frac{1}{2 I_{d}^{\dagger}}\left(p_{\theta_{12}}^{2}+\frac{p_{\phi_{12}}^{2}}{\sin ^{2} \theta_{12}}\right)+\sum_{i=1}^{2}\left\{\frac{1}{2 I_{A_{i}}^{\dagger} \sin ^{2} \theta_{i}}\left[\left(p_{\phi_{i}}-p_{\psi_{i}} \cos \theta_{i}\right) \cos \psi_{i}-p_{\theta_{i}} \sin \theta_{i} \sin \psi_{i}\right]^{2}\right. \\
& \left.+\frac{1}{2 I_{B_{i}}^{\dagger} \sin ^{2} \theta_{i}}\left[\left(p_{\phi_{i}}-p_{\psi_{i}} \cos \theta_{i}\right) \sin \psi_{i}+p_{\theta_{i}} \sin \theta_{i} \cos \psi_{i}\right]^{2}+\frac{1}{2 I_{C_{i}}^{\dagger}} p_{\psi_{i}}^{2}\right\}+V\left(R^{\dagger}, \theta_{12}, \phi_{12}, \boldsymbol{\Theta}, \boldsymbol{\Phi}, \Psi\right)
\end{aligned}
$$

where $\boldsymbol{\theta} \equiv\left(\theta_{1}, \theta_{2}\right), \boldsymbol{\Phi} \equiv\left(\phi_{1}, \phi_{2}\right), \Psi \equiv\left(\psi_{1}, \psi_{2}\right)$, and $V\left(R^{\dagger}\right.$, $\left.\theta_{12}, \phi_{12}, \boldsymbol{\Theta}, \boldsymbol{\Phi}, \Psi\right)$ is the potential energy for the given separation $R^{\dagger}$, and orientation of the rigid rotor fragments. $I_{d}^{\dagger}$ is the "diatomic" moment of inertia for the centers of mass of the two fragments and equals $\mu_{12} R^{\dagger^{2}} . I_{A_{i}}^{+}, I_{B_{i}}^{+}$, and $I_{C_{i}}^{+}$are the principal moments of inertia of the rigid body fragments with their transition state structure.

Evaluation of the integral over the momenta yields

$$
\begin{aligned}
Q_{t}^{\dagger}= & \frac{1}{\sigma^{\dagger}} \frac{2 \pi I_{d}^{\dagger} k_{B} T}{h^{2}}\left\{\prod_{i=1}^{2}\left[8 \pi^{3} I_{A_{i}}^{\dagger} I_{B_{i}}^{\dagger} I_{C_{i}}^{\dagger}\left(k_{B} T / h^{2}\right)^{3}\right]^{1 / 2}\right\} \\
& \times \int d \theta_{12} d \phi_{12} \sin \theta_{12}\left(\prod_{i=1}^{2} d \theta_{i} d \phi_{i} d \psi_{i} \sin \theta_{i}\right) \\
& \times \exp \left[-\beta V\left(R^{\dagger}, \theta_{12}, \phi_{12}, \Theta, \Phi, \Psi\right)\right] .
\end{aligned}
$$

Upon integration over the $\theta_{i}, \phi_{i}$, and $\psi_{\mathrm{i}}$ for a fixed $\theta_{12}$ and $\phi_{12}$, the result, by symmetry, is independent of the actual 
orientation in space, $\theta_{12}$ and $\phi_{12}$, of the two fragments, and so the integral over $\theta_{12}$ and $\phi_{12}$ may be immediately evaluated to give

$$
Q_{t}^{\dagger}=\frac{1}{\sigma^{\dagger}} Q_{t, d}^{\dagger} Q_{i, h r}^{\dagger}
$$

where $Q_{t, d}^{\dagger}$ is the usual classical diatomic partition function $2 I_{d}^{\dagger} k_{B} T / \hbar^{2}$, and

$$
\begin{aligned}
Q_{t, h r}^{\dagger}= & \left\{\prod_{i=1}^{2}\left[8 \pi^{3} I_{A_{i}}^{\dagger} I_{B_{i}}^{\dagger} I_{C_{i}}^{\dagger}\left(k_{B} T / h^{2}\right)^{3}\right]^{1 / 2}\right\} \\
& \times \int\left(\prod_{i=1}^{2} d \theta_{i} d \phi_{i} d \psi_{i} \sin \theta_{i}\right) \\
& \times \exp \left[-\beta V\left(R^{\dagger}, 0,0, \boldsymbol{\Theta}, \boldsymbol{\Phi}, \Psi\right)\right],
\end{aligned}
$$

with $\theta_{12}$ and $\phi_{12}$ having been set equal to zero.

For the case of a reasonably loose transition state $Q_{t, h r}^{+}$ can be evaluated via crude Monte Carlo integration. ${ }^{35}$ For a tight transition state many of the configurations sampled will not contribute to the integral and one might revert to the calculation method of Wardlaw and Marcus ${ }^{4-7}$ or else use some form of stratified sampling such as that discussed by Farantos et al. ${ }^{11}$ for microcanonical RRKM determinations. In the present application to the methyl radical recombination reaction crude Monte Carlo integration sufficed. The final Monte Carlo result for the rate constant for the case of free radical recombination is

$k_{\infty}^{C l}(T)$

$$
\begin{aligned}
= & g_{e} \frac{\sigma_{1} \sigma_{2}}{\sigma^{\dagger}} R^{+2}\left(\frac{8 \pi k_{B} T}{\mu_{12}}\right)^{1 / 2}\left\{\prod_{i=1}^{N_{v}} \frac{\sinh \left(\frac{h v_{i}}{2 k_{B} T}\right)}{\sinh \left(\frac{h v_{i}^{\dagger}}{2 k_{B} T}\right)}\right\} \\
& \times\left(\prod_{i=1}^{2} \frac{I_{A_{i}}^{\dagger} I_{B_{i}}^{\dagger} I_{C_{i}}^{\dagger}}{I_{A_{i}} I_{B_{i}} I_{C_{i}}}\right)^{1 / 2} \frac{1}{N_{\mathrm{MC}}} \sum_{i=1}^{N_{\mathrm{MC}}} \exp \left[-\beta V_{i}\right],
\end{aligned}
$$

where $N_{v}$ is the number of conserved vibrational modes for the two fragments, and $v_{i}$ and $v_{i}^{\dagger}$ are the vibration frequencies for the $i$ th conserved mode of the reactants and of the transition state, respectively. $V_{i}$ is the value of the potential for the $i$ th randomly selected configuration, and $N_{\mathrm{MC}}$ is the number of random points.

\section{DETERMINATION OF THE QUANTUM PARTITION FUNCTION FOR THE TRANSITIONAL MODES}

The primitive $P$-point discretization of the path integral expression for the quantum transitional mode partition function is given by

$$
\begin{aligned}
Q_{t}^{\dagger}= & \int d \mathbf{\Lambda}^{1} \cdots d \Lambda^{P} d \mathbf{\Omega}^{1} \cdots d \mathbf{\Omega}^{P} \\
& \times \prod_{s=1}^{P} \rho_{0}\left(\Lambda^{s+1}, \Lambda^{s} ; \beta / P\right) \rho_{0}\left(\mathbf{\Omega}^{s+1}, \mathbf{\Omega}^{s} ; \beta / P\right) \\
& \times \exp \left[-\frac{\beta}{P} V\left(\Lambda^{s}, \mathbf{\Omega}^{s}\right)\right],
\end{aligned}
$$

where $\boldsymbol{\Lambda}^{s}$ refers to the line of centers orientations

$$
\Lambda^{s}=\left(\cos \theta_{12}^{s}, \phi_{12}^{s}\right),
$$

and $\boldsymbol{\Omega}^{s}$ refers to the Euler angles

$$
\mathbf{\Omega}^{s}=\prod_{i=1}^{2} \boldsymbol{\Omega}_{i}^{s}=\prod_{i=1}^{2}\left(\cos \theta_{i}^{s}, \phi_{i}^{s}, \psi_{i}^{s}\right) .
$$

In Eq. (3.1), $\boldsymbol{\Lambda}^{P+1}=\boldsymbol{\Lambda}^{1}$ and $\boldsymbol{\Omega}^{P+1}=\boldsymbol{\Omega}^{1}$, while $\rho_{0}\left(\Lambda^{s+1}, \Lambda_{;}^{s} ; \beta / P\right)$ and $\rho_{0}\left(\boldsymbol{\Omega}^{s+1}, \boldsymbol{\Omega}^{s} ; \beta / P\right)$, the "free density matrices," are the density matrices for the free-rotor system at a temperature $P T$. [The latter has $V(\Lambda, \Omega) \equiv 0$.]

In the present treatment the quantity of interest is the combined partition function for the hindered rotation of the two separate fragments and for the rotation of the line of centers. If the integrals over $\boldsymbol{\Omega}^{s}$ at fixed line of centers orientations $\boldsymbol{\Lambda}^{s}$ are performed first, the resulting expression is independent of $\boldsymbol{\Lambda}^{s}$ because there is no external field acting on the reacting pair. Thus, the integrals over the $\Lambda^{s}$ may be evaluated separately to give the partition function for the rotation of a diatomic molecule with point masses equal to those of the two fragments. Expressions for the remaining partition function for the hindered rotations are now easily obtained from the analogous expressions derived by $\mathrm{Ku}$ harski and Rossky, ${ }^{36}$ who utilized some results of Schul$\operatorname{man}^{37}$ and introduced a short-time ("fixed-axis") method ${ }^{38}$ to determine the quantum partition function for the hindered translational and hindered rotational degress of freedom of liquid $\mathrm{H}_{2} \mathrm{O}$ and $\mathrm{D}_{2} \mathrm{O}$. A brief review of their derivations, with application to the present two fragment case, is presented below for clarity.

The quantum partition function may be expressed as in Eq. (2.7) except that now $Q_{t, d}^{\dagger}$ and $Q_{t, h r}^{\dagger}$ are to be evaluated quantum mechanically. In particular,

$$
\begin{aligned}
Q_{t, h r}^{\dagger}= & \int d \mathbf{\Omega}^{1} \cdots d \mathbf{\Omega}^{P} \prod_{s=1}^{P} \rho_{0}\left(\mathbf{\Omega}^{s+1}, \mathbf{\Omega}^{s} ; \beta / P\right) \\
& \times \exp \left[-\frac{\beta}{P} V\left(\mathbf{\Omega}^{s}\right)\right],
\end{aligned}
$$

and $Q_{t, d}^{\dagger}$, the quantum diatomic partition function, is given (for $T>2 I_{d}^{\dagger} k_{B} / \hbar^{2} \equiv \Theta_{r}^{-1}$ ) by the expansion ${ }^{39}$

$$
\begin{aligned}
Q_{t, d}^{\dagger}= & \frac{T}{\Theta_{r}}\left[1+\frac{1}{3}\left(\frac{\Theta_{r}}{T}\right)+\frac{1}{15}\left(\frac{\Theta_{r}}{T}\right)^{2}\right. \\
& \left.+\frac{4}{315}\left(\frac{\Theta_{r}}{T}\right)^{3}+\cdots\right] .
\end{aligned}
$$

Using the least action approximation to the sum over all classical paths for the transition from the orientation $\boldsymbol{\Omega}^{s}$ to the orientation $\Omega^{s+1}$ in the time period $\beta \hbar / P$ gives the free rotation matrix as ${ }^{37}$

$$
\begin{aligned}
\rho_{0}\left(\mathbf{\Omega}^{s+1}, \mathbf{\Omega}^{s} ; \beta / P\right)= & C \prod_{i=1}^{2}\left[D\left(\boldsymbol{\Omega}_{i}^{s+1}, \mathbf{\Omega}_{i}^{s} ; \beta / P\right)\right]^{1 / 2} \\
& \times \exp \left[-S_{\mathrm{Cl}}\left(\mathbf{\Omega}_{i}^{s+1}, \mathbf{\Omega}_{i}^{s} ; \beta / P\right) / \hbar\right],
\end{aligned}
$$

where $C$ is a constant, $D\left(\Omega_{i}^{s+1}, \Omega_{i}^{s} ; \beta / P\right)$ is the Van Vleck determinant ${ }^{40}$ associated with the transition from $\boldsymbol{\Omega}_{i}^{s}$ to $\boldsymbol{\Omega}_{i}^{s+1}$ in a time period of $\beta \hbar / P$, and $S_{\mathrm{Cl}}\left(\boldsymbol{\Omega}_{i}^{s+1}, \boldsymbol{\Omega}_{i}^{s} ; \beta / P\right)$ is the action along the least action classical path between the two orientations. In the exact free rotation matrix there are 
contributions from multiple rotations. However, for sufficiently large $P$ ("sufficiently small times $\beta / P$ ") these contributions are negligible, and, in the limit of large enough $P$, Eq. (3.6) becomes exact.

A fixed-axis approximation is next introduced to determine the Van Vleck determinant and the classical action. ${ }^{36}$ This fixed-axis approximation, which is valid for short times and is thus accurate at large $\boldsymbol{P}$ or high temperature, yields ${ }^{36}$

$$
D_{F A}\left(\Omega_{i}^{s+1}, \mathbf{\Omega}_{i}^{s} ; \beta \hbar / P\right)=\frac{I_{A_{i}}^{\dagger} I_{B_{i}}^{\dagger} I_{C_{i}}^{\dagger}}{(\beta \hbar / P)^{3}}\left[\frac{\Gamma_{i}^{s+1, s} / 2}{\sin \left(\Gamma_{i}^{s+1, s / 2)}\right.}\right]^{2},
$$

where $\Gamma_{i}^{s+1, s}$, the arc length of the rotation from orientation $s$ to orientation $s+1$ of fragment $i$, is given by ${ }^{36}$

$$
\Gamma_{i}^{s+1, s}=2 \cos ^{-1}\left(\chi_{i}^{s+1, s}\right),
$$

and $\chi_{i}^{s+1, s}$ is a quaternion parameter for the rotation from orientation $\boldsymbol{\Omega}_{i}^{s}$ to orientation $\boldsymbol{\Omega}_{i}^{s+1}$, the four of which, in general, are given in terms of Euler angles as ${ }^{41,42}$

$$
\begin{array}{ll}
\chi=\cos \frac{\theta}{2} \cos \frac{(\psi+\phi)}{2}, & \eta=\sin \frac{\theta}{2} \cos \frac{(\psi-\phi)}{2}, \\
\xi=\sin \frac{\theta}{2} \sin \frac{(\psi-\phi)}{2}, \quad \xi=\cos \frac{\theta}{2} \sin \frac{(\psi+\phi)}{2} .
\end{array}
$$

The use of these quaternion parameters provides a convenient set of parameters to describe the orientation after a rotation has been performed on some initial orientation, and they also provide a convenient method for determining the relative orientation of two orientations. (See, e.g., Refs. 41 and 42.)

The least classical action for the rotation from orientation $s$ to orientation $s+1$ of fragment $i$, in the fixed-axis approximation, is given by

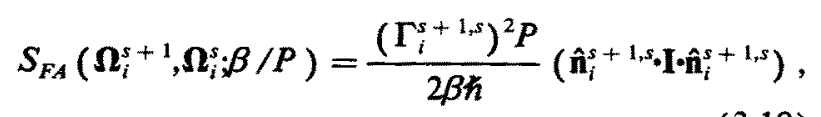

where, using the quaternions, the rotation axis $\hat{\mathbf{n}}_{i}^{s+1, s}$ is given in the prinicpal axis frame by ${ }^{36}$

$$
\hat{\mathbf{n}}_{i}^{s+1, s}=\frac{1}{\sin \left(\Gamma_{i}^{s+1, s} / 2\right)}\left(\eta_{i}^{s+1, \hat{i}_{i}}-\xi_{i}^{s+1, \hat{j}_{j}}+\zeta_{i}^{s+1, s \hat{k}}\right),
$$

and the last term in parenthesis in Eq. (3.10) is a tensor product, I being the moment of inertia tensor in the principal axis frame. Equation (3.10) has the form which parallels the usual one for a free translational coordinate: a distance squared (here an arc length squared), multiplied by a mass (here a moment of inertia), and divided by twice a time, $\beta \hbar$ / $P$.

The final expression for the quantum partition function for the hindered rotation of two rigid rotor molecules is thus

$$
\begin{aligned}
Q_{t, h r_{Q}}^{\dagger}= & C\left[\prod_{i=1}^{2} \frac{I_{A_{i}}^{\dagger} I_{B_{i}}^{\dagger} I_{C_{i}}^{\dagger}}{(\beta \hbar / P)^{3}}\right]^{1 / 2} \\
& \times \int d \mathbf{\Omega}^{1} \cdots d \mathbf{\Omega}^{P} \prod_{s=1}^{P}\left\{\prod_{i=1}^{2} \frac{\Gamma_{i}^{s+1, s / 2}}{\sin \left(\Gamma_{i}^{s+1, s / 2} / 2\right.}\right. \\
& \left.\times \exp \left[-\frac{1}{\hbar} S_{F A}\left(\mathbf{\Omega}_{i}^{s+1}, \mathbf{\Omega}_{i}^{s} ; \frac{\beta \hbar}{P}\right)\right]\right\} \\
& \times \exp \left[-\frac{\beta}{P} V\left(\mathbf{\Omega}^{s}\right)\right]
\end{aligned}
$$

As discussed by Doll and Myers, ${ }^{43}$ quantum partition functions may be obtained from Monte Carlo evaluation of an appropriate partition function ratio. In the present article two alternative partition function ratios were used to determine the quantum partition function. One alternative involves the determination of the ratio of the quantum partition function relative to that for two free rotors:

$$
\frac{Q_{i, k r_{Q}}^{\dagger}}{Q_{i, f r_{Q}}^{\dagger}}=\frac{\int d \Gamma A \exp \left[-(\beta / P) \Sigma_{s=1}^{P} V\left(\Omega^{s}\right)\right]}{\int d \Gamma A},
$$

where

$$
\begin{aligned}
& Q_{i, r_{Q}}^{\dagger}=\int d \Gamma A, \\
& d \Gamma=d \mathbf{\Omega}^{1} \cdots d \mathbf{\Omega}^{P},
\end{aligned}
$$

and

$$
A=\prod_{s=1}^{P} \rho_{0}\left(\boldsymbol{\Omega}^{s+1}, \mathbf{\Omega}^{s} ; \beta / P\right) .
$$

The quantum partition function for the free rotation of the two fragments in their transition state configuration, $Q_{i, r r_{e}}^{\dagger}$, is given by the high temperature expansion ${ }^{44}$

$$
Q_{i, r_{Q}}^{\dagger}=Q_{i, f r_{\mathrm{Cl}}}^{\dagger} \prod_{i=1}^{2}\left[1+\frac{\hbar^{2}}{24 k_{B} T} \sum_{\text {cyclic }}\left(\frac{2}{I_{A_{i}}^{\dagger}}-\frac{I_{A_{i}}^{\dagger}}{I_{B_{i}}^{\dagger} I_{C_{i}}^{\dagger}}\right)\right] \text {, }
$$

where

$$
Q_{i, r_{\mathrm{CC}}}^{\dagger}=\prod_{i=1}^{2} 8 \pi^{2}\left[8 \pi^{3} I_{A_{i}}^{\dagger} I_{B_{i}}^{\dagger} I_{C_{i}}^{\dagger}\right]^{1 / 2}\left(k_{B} T / h^{2}\right)^{3 / 2}
$$

Thus, $Q_{i, h r_{Q}}^{\dagger}$ may be obtained from Eqs. (3.13)-(3.16) with Monte Carlo evaluation of the ratio given in Eq. (3.13). This alternative is once again restricted to reasonably loose transition states.

The other alternative is to calculate the ratio of the quantum to the classical partition function. The classical partition function for the hindered rotor degrees of freedom may be written as

$$
Q_{t, h r_{\mathrm{C}}}^{\dagger}=Q_{t, r_{\mathrm{C}}}^{\dagger} \frac{\int d \mathbf{\Omega}^{1} \exp \left[-\beta V\left(\boldsymbol{\Omega}^{1}\right)\right]}{\int d \mathbf{\Omega}^{1}}
$$

Multiplying both sides of Eq. (3.17) by $Q_{t, f r_{Q}}^{\dagger} / Q_{t, r_{\mathrm{Cl}}}^{\dagger}$, and using Eq. (3.14a), yields

$$
Q_{i, h r_{\mathrm{Cl}}}^{\dagger} \frac{Q_{i, r_{Q}}^{\dagger}}{Q_{i, f r_{\mathrm{C}}}^{\dagger}}=\frac{\int d \Gamma A \int d \Omega^{1} \exp \left[-\beta V\left(\Omega^{1}\right)\right]}{\int d \mathbf{\Omega}^{1}},
$$


where $d \Gamma$ and $A$ are given by Eqs. (3.14b) and (3.14c), respectively. Since $\int A d \mathbf{\Omega}^{2} \cdots d \mathbf{\Omega}^{P}$ is independent of $\Omega^{1}, \int d \boldsymbol{\Gamma} A$ can be written as $\left(\int d \mathbf{\Omega}^{1}\right)\left(\int A d \mathbf{\Omega}^{2} \cdots d \mathbf{\Omega}^{P}\right)$, and hence Eq. (3.18) may be written as

$$
Q_{t, h r_{\mathrm{Cl}}}^{\dagger} \frac{Q_{t, f r_{Q}}^{\dagger}}{Q_{i, r_{\mathrm{Cl}}}^{\dagger}}=\int d \Gamma A \exp \left[-\beta V\left(\Omega^{1}\right)\right] .
$$

Thus, using Eqs. (3.13), (3.14a), and (3.19), the ratio of the quantum to classical partition functions for the transitional modes may be written as

$$
\frac{Q_{t, h r_{Q}}^{\dagger}}{Q_{t, h r_{C l}}^{\dagger}}=\frac{Q_{t, f r_{Q}}^{\dagger}}{Q_{t, f r_{C l}}^{\dagger}} \frac{\int d \Gamma A \exp \left[-(\beta / P) \Sigma_{s=1}^{P} V\left(\mathbf{\Omega}^{s}\right)\right]}{\int d \Gamma A \exp \left[-\beta V\left(\mathbf{\Omega}^{1}\right)\right]} .
$$

The Monte Carlo calculation of this ratio is feasible regardless of how tight or loose the transition state is. On the other hand, the determination of $k_{\infty}^{Q}(T)$ (the quantum free radical recombination rate constant) from the $Q_{t, h r_{Q}}^{\dagger}$ in Eq. (3.13) requires only a single Monte Carlo integration, rather than, as in Eq. (3.20), the Monte Carlo evaluation of both the ratio $Q_{t, h r_{Q}}^{\dagger} / Q_{t, h r_{\mathrm{C}}}^{\dagger}$ and the quantity $Q_{t, h r_{\mathrm{Cl}}}^{\dagger}$.

Importance sampling techniques ${ }^{4,46}$ may now be used together with the fixed-axis approximation for $A$ discussed above, in the Monte Carlo evaluation of the path integral ratios given in Eqs. (3.13) and (3.20). In particular, in the evaluation of Eq. (3.20) a weighting factor

$$
W_{j}=A \exp \left[-\frac{\beta}{P} V\left(\Omega^{1}\right)\right],
$$

with $A$ given by Eq. (3.14c), is used to determine whether to accept or reject each random step generated in accordance with the parameters described in the next section.

The integrand in Eq. (3.20) which is summed up is then

$$
Y_{j}=\exp \left\{-\frac{\beta}{P}\left[\sum_{s=1}^{P} V\left(\Omega^{s}\right)-V\left(\Omega^{1}\right) P\right]\right\},
$$

with the quantum to classical ratio being given by

$$
\frac{Q_{i, h r_{Q}}^{\dagger}}{Q_{t, h r_{\mathrm{Cl}}}^{\dagger}}=\frac{Q_{t, r_{Q}}^{\dagger}}{Q_{t, f r_{\mathrm{Cl}}}^{\dagger}} \sum_{j=1}^{N_{\mathrm{MC}}} Y_{j}
$$

Multiplying the result of this Monte Carlo evaluation of Eq. (3.13) by the quantum correction factor for the diatomic term $Q_{t, d}^{\dagger}$ [given by the quantity in brackets in Eq. (3.5)] and then dividing by the quantum correction factor for $Q_{i}^{r}$ [given by the term multiplying $Q_{t, j r_{\mathrm{Cl}}}^{\dagger}$ in Eq. (3.15), with the transition state moments of inertia replaced by those for the reactant configuration ] yields the ratio $k_{\infty}^{Q}(T) / k_{\infty}^{\mathrm{Cl}}(T)$.

For the quantum to free-rotor ratio given in Eq. (3.13) the weighting factor is, instead,

$$
W_{j}=A \text {, }
$$

and the integrand in Eq. (3.13) is now given by

$$
Y_{j}=\exp \left[-\frac{\beta}{P} \sum_{s=1}^{P} V\left(\Omega^{s}\right)\right] .
$$

The result of this Monte Carlo evaluation of Eq. (3.13) is next multiplied by the quantum expression for $Q_{t, d}^{\dagger}$, given by Eq. (3.5), to give the quantum result for $Q_{?}^{\dagger}$. Substituting this result into Eq. (2.3) and inserting the quantum correction factor for $Q_{t}^{r}$ yields the quantum free radical recombination rate constant $k_{\infty}^{Q}(T)$.

\section{RESULTS AND DISCUSSION}

The two formalisms described above were implemented for the methyl radical recombination reaction using the potential energy function employed by Wardlaw and Marcus. $^{5,6}$ The structure of the rigid body fragments for a given $R^{\dagger}$ were also taken to be as in Refs. 5, and 6. $g_{e}, \sigma_{\mathrm{CH}_{3}}$, and $\sigma^{\dagger}$ were taken to be $1 / 4,6$, and 72 , respectively.

For the evaluation of the quantum to free-rotor ratio the following procedure was followed: (1) One of the two fragments $i$ was chosen randomly and an absolute orientation in space, which we will denote by $s=0$, was chosen randomly. ${ }^{47}$ The subsequent points [the path integral points in Eq. (3.1) ] correspond to $s=1, \ldots, P$. The molecule corresponding to fragment $i$, and path integral discretization point $s$ will be denoted as $(i, s)$, and the molecule corresponding to the $s=0$ point is denoted by $(i, 0)$. For this method, this orientation has no effect on the weighting factor and so was always accepted. (2) Next, one of the discretization points $s$ was chosen randomly, and then a rotation about a randomly chosen axis $^{48}$ of the old orientation of molecule $(i, s)$ relative to molecule $(i, 0)$ was performed. The magnitude of the rotation was chosen as $R \delta_{1}^{f r}$, where $R$ is a random number between 0 and 1 , and $\delta_{1}^{f r}$, the maximum magnitude of the rotation, was chosen to give a roughly $50 \%$ acceptance ratio $^{49}$ of the rotations. (3) The new orientation of molecule $(i, s)$ relative to the zeroth order orientation $(i, 0)$ was then calculated using the formula for the composition of quaternions given in Eq. (33) of Ref. 41. (4) The new orientation of molecule $(i, s)$ relative to that of $(i, s+1)$, and that of $(i, s-1)$ was next calculated from the known orientations of $(i, s),(i, s+1)$, and $(i, s-1)$ relative to that of $(i, 0)$, using the formula for relative quaternions given by Eq. (38) of Ref. 41. (5) The ratio of the weighting factor $W_{j}$, given in Eq. (3.24), for this new orientation relative to $W_{j}$ for the old orientation was calculated. If this number was greater than a random number between 0 and 1 then the rotation was accepted. Otherwise the rotation in step (2) was rejected and the old relative orientations of $(i, s)$ to $(i, 0),(i, s)$ to $(i, s+1)$, and $(i, s)$ to $(i, s-1)$ were restored to their old values. (6) The absolute orientation in space of molecule $(i, s)$ was then calculated, from Eq. (33) of Ref. 41 for the composition of quaternions, using the orientation of molecule $(i, s)$ relative to that of molecule $(i, 0)$ and the absolute orientation in space of molecule $(i, 0)$. The new value of the integrand was then calculated from Eq. (3.25) using the new absolute orientation in space of molecule $(i, s)$. This process was then repeated until the desired number of Monte Carlo points was reached.

The evaluation of the quantum to classical ratio was calculated in a similar manner except that now the weighting factor $W_{j}$ given in Eq. (3.21) is used. In this case $W_{j}$ does depend on the "zeroth" order orientation so that step (1) now involves a rotation of random magnitude, within a maximum magnitude $\delta_{0}^{\mathrm{Cl}}$, of the zeroth order orientation about a randomly chosen axis. ${ }^{48}$ The maximum magnitude of the 
rotation here was much larger than that of step (2) (labeled $\delta_{1}^{\text {Cl }}$ ) in order to allow for an efficient sampling over configuration space, as discussed by Kuharski and Rossky. ${ }^{36}$ The other steps were identical to those in the quantum to freerotor case, except in the present case the old $(i, 0)$ absolute orientation also had to be restored in step (5) whenever a move was rejected. The overall acceptance ratio was once again kept at about $50 \%{ }^{49}$ by varying the parameters $\delta_{0}^{\mathrm{CI}}$ and $\delta_{1}^{\mathrm{Cl}}$.

All of the calculations discussed here were easily programmed and large computation times were not required. ${ }^{50}$ The results for $k_{\infty}^{\mathrm{Cl}}(T), k_{\infty}^{Q}(T)$, and the ratio $k_{\infty}^{Q}(T) / k_{\infty}^{\mathrm{Cl}}(T)$ obtained from these calculations are given in Table I for the four temperatures $300,500,1000$, and 2000 $K$. All of the quantum results reported in Table I were calculated using $P=6$ discretization points, since it was found that the results had converged by this value of $P .{ }^{51}$ In the classical calculations the estimated error, $\sigma^{\mathrm{MC}}$, was evaluated from

$$
\sigma^{\mathrm{MC}}=\left[\left|f^{2}-\sum_{i=1}^{N_{\mathrm{MC}}} f_{i}^{2} / N_{\mathrm{MC}}\right| / N_{\mathrm{MC}}\right]^{1 / 2},
$$

where $f$ is the Monte Carlo result for the quantity being evaluated [in the present case $k_{\infty}^{\mathrm{Cl}}(T)$ ] and $f_{i}$ is a particular value of the integrand. In the quantum calculations, due to the strong correlation between successive states, $\sigma^{\mathrm{MC}}$ was instead calculated from the variance of sequences of estimates to $f\left[f\right.$ being either $k_{\infty}^{Q}(T)$ or the ratio $\left.k_{\infty}^{Q}(T) / k_{\infty}^{\mathrm{Cl}}(T)\right]$ over blocks of 100 to 1000 Monte Carlo steps. (See, e.g., Ref. 46.)

Comparison of the results for $k_{\infty}^{\mathrm{Cl}}(T)$ given in Table I, with those given in Table VII of Ref. 6, shows that within the Monte Carlo error bars the two calculations give the same result. One also sees from Table I that, within the Monte Carlo error bars, the quantum correction for recombination in the present case is negligible, and is at most about $1 \%$ to $2 \%$, and its temperature dependence is, within the statistical error, also negligible. At low temperatures one might expect that there would be a larger quantum correction for these modes. However, when the temperature is reduced the loca-
TABLE I. Quantum corrections from path integration.

\begin{tabular}{rcccc}
\hline $\begin{array}{c}\text { Temp } \\
(\mathrm{K})\end{array}$ & $\begin{array}{c}\mathbf{R}^{+\mathrm{a}} \\
(\AA)\end{array}$ & $\begin{array}{c}k_{\infty}^{\mathrm{Cl}}(T)^{\mathrm{b}} \pm \sigma_{\mathrm{MC}}^{\mathrm{c}}{ }^{\mathrm{k}} k_{\infty}^{Q}(T)^{\mathrm{d}} \pm \sigma_{\mathrm{MC}}{ }^{\mathrm{c}} \\
\left(10^{13} \mathrm{~cm}^{3} \mathrm{~mol}^{-1}\right)\left(10^{13} \mathrm{~cm}^{3} \mathrm{~mol}^{-1}\right)\end{array}$ & Ratio $^{\mathrm{c}} \pm \sigma_{\mathrm{MC}}{ }^{\mathrm{c}}$ \\
\hline 300 & 4.2 & $5.06 \pm 0.02$ & $4.99 \pm 0.02$ & $1.002 \pm 0.004$ \\
500 & 3.8 & $4.32 \pm 0.03$ & $4.31 \pm 0.04$ & $0.999 \pm 0.006$ \\
1000 & 3.4 & $2.83 \pm 0.03$ & $2.75 \pm 0.04$ & $0.997 \pm 0.007$ \\
2000 & 3.0 & $1.38 \pm 0.02$ & $1.34 \pm 0.03$ & $1.024 \pm 0.008$ \\
\hline
\end{tabular}

${ }^{a}$ As determined in Ref. 6.

${ }^{b}$ As determined by the present classical Monte Carlo calculation.

${ }^{c} \sigma_{M C}$ refers to the Monte Carlo uncertainties determined as discussed in the text.

As determined by the quantum to free-rotor ratio path integral ratio calculation. [See Eq. (3.13).]

${ }^{e} k_{\infty}^{Q} / k_{\infty}^{\mathrm{Cl}}$ as determined by the quantum to classical ratio path integral calculation. [See Eq. (3.20).]

tion of the transition state moves to larger separation distances and so the partition function for the transitional modes becomes more and more like the product of free-rotor partition functions for which the quantum correction is relatively minor (except at quite low temperatures in which case one expects the quantum corrections for the transitional modes of the transition state and for the transitional modes of the reactants to cancel). Conversely, at high temperatures one might expect a quantum correction due to the decrease in $R^{\dagger}$, causing an increase in the steepness of the potential energy function. That this is not the case here must be because the increase in temperature causing more classical behavior balances out the increase in steepness causing quantum effects. In other systems which are "tighter" in the transition state region (have stronger fragment-fragment bending forces there) there may be larger quantum effects and the present method may be used to determine the quantum correction.

It is interesting to compare these results with those which can be obtained from a generalized Wigner-Kirkwood expansion of the partition function. ${ }^{52,53}$ For the present system of two symmetric tops plus a linear rigid rotor this expansion of the partition function is given by ${ }^{52,53}$

$$
\frac{Q_{t, h r_{Q}}^{\dagger}}{Q_{t, h r_{\mathrm{Cl}}}^{\dagger}}=\frac{Q_{t, f r_{Q}}^{\dagger}}{Q_{t, f r_{\mathrm{Cl}}}^{\dagger}} \frac{\int d \theta_{12} d \phi_{12} \Pi_{i=1}^{2} d \theta_{i} d \phi_{i} d \psi_{i} \exp (-\beta V)\left[1-\beta^{3} \hbar^{2} \Sigma_{k}\left(\nabla_{k} V\right)^{2} / 24 m_{k}\right]}{\int d \theta_{12} d \phi_{12} \Pi_{i=1}^{2} d \theta_{i} d \phi_{i} d \psi_{i} \exp (-\beta V)},
$$

where

$$
\begin{aligned}
\frac{1}{m_{k}}\left(\nabla_{k} V\right)^{2}= & \frac{1}{I_{d}^{\dagger}}\left[\left(\frac{\partial}{\partial \theta_{12}} V\right)^{2}+\frac{1}{\sin ^{2} \theta_{12}}\left(\frac{\partial}{\partial \phi} V\right)^{2}\right] \\
& +\sum_{i=1}^{2}\left[\frac{1}{I_{A_{i}}^{\dagger}}\left(\frac{\partial}{\partial \theta_{i}} V\right)^{2}+\frac{1}{I_{A_{i}}^{\dagger} \sin ^{2} \theta_{i}}\left(\frac{\partial}{\partial \phi_{i}} V-\frac{\partial}{\partial \psi_{i}} V\right)^{2}+\frac{1}{I_{C_{i}}^{\dagger}}\left(\frac{\partial}{\partial \psi_{i}} V\right)^{2}\right] .
\end{aligned}
$$

The derivation of the Wigner-Kirkwood expansion for symmetric tops and for diatomic rotors has been given previously (see, e.g., Refs. 54 and 55).

Results based on Eqs. (4.2) and (4.3) are presented in Table II. The Monte Carlo evaluations in the calculation of $k_{\infty}^{Q}(T)$ have been performed in exact analogy to the classical calculations of Table $I$. The ratio calculations have been performed along the lines of the quantum calculations in Table I. In both cases the derivatives given in Eq. (4.3) were calculated numerically, and, as a result of the extra potential calls thus required, the evaluation of Eq. (4.2) required more CPU time than the exact calculations. However, this would not be the case if the potential was given in a form which was easily differentiated analytically. Comparison of 
TABLE II. Quantum corrections from generalized Wigner-Kirkwood expansion.

\begin{tabular}{rlll}
\hline $\begin{array}{c}\text { Temp } \\
(\mathrm{K})\end{array}$ & $\begin{array}{l}R^{\text {ta }} \\
(\AA)\end{array}$ & $\begin{array}{c}k_{\infty}^{Q}(T)^{\mathrm{b}} \pm \sigma_{\mathrm{MC}}^{\mathrm{c}} \\
\left(10^{13} \mathrm{~cm}^{3} \mathrm{~mol}^{-1}\right)\end{array}$ & Ratio $^{\mathrm{d}} \pm \sigma_{\mathrm{MC}}{ }^{\mathrm{c}}$ \\
\hline 300 & 4.2 & $5.00 \pm 0.03$ & $0.990 \pm 0.001$ \\
500 & 3.8 & $4.22 \pm 0.05$ & $0.980 \pm 0.001$ \\
1000 & 3.4 & $2.70 \pm 0.06$ & $0.980 \pm 0.001$ \\
2000 & 3.0 & $1.29 \pm 0.05$ & $0.982 \pm 0.001$ \\
\hline
\end{tabular}

${ }^{2}$ As determined in Ref. 6.

${ }^{b}$ Determined by the direct evaluation of $k_{\infty}^{Q}(T)$ from the generalized Wigner-Kirkwood expansion.

${ }^{c} \sigma_{\mathrm{MC}}$ refers to the Monte Carlo uncertainties determined as discussed in the text.

${ }^{d}$ Determined by the direct evaluation of $k_{\infty}^{Q} / k_{\infty}^{\mathrm{Cl}}$ from the generalized Wigner-Kirkwood expansion calculation. The Monte Carlo error bars in this calculation are negligible.

the results given for $k_{\infty}^{Q}(T)$ in Tables I and II shows that the Wigner-Kirkwood result is approximately within the error bars of the nonperturbative result. The smallness of $\sigma_{\mathrm{MC}}$ in the last column of Table II is presumably because in that calculation of a ratio $k_{\infty}^{Q}(T) / k_{\infty}^{\mathrm{Cl}}(T)$ only a small correction term had to be averaged.

Although the results given in Table I indicate a very small correction for the transitional modes of a relatively loose transition state at reasonable temperatures, there may still be a large correction to some of the $k_{E J}$ 's at lower energies. For some experimental situations quantities of particular interest are these $k_{E J}$ 's, and, for this reason, we are presently studying the possibility of obtaining accurate microcanonical correction factors. When only microcanonical rate constants $k_{E}$ are needed, they can be obtained by inverse Laplace transformation of results at fixed $R$, provided one evaluates $k_{\infty, Q}(T)$ over a wide enough range of temperatures. However, the determination of the constant $E$ and $J$ rate constants requires a different approach.

\section{ACKNOWLEDGMENTS}

It is a pleasure to acknowledge support of this research by the National Science Foundation. S. J. K. gratefully acknowledges the support of a Natural Sciences and Engineering Research Council of Canada Postgraduate Scholarship, 1984-1987.

'P. J. Robinson and K. A. Holbrook, Unimolecular Reactions (Wiley, New York, 1972); W. Forst, Theory of Unimolecular Reactions (Academic, New York, 1973).

${ }^{2}$ R. E. Weston and H. A. Schwarz, Chemical Kinetics (Prentice-Hall, Englewood Cliffs, 1972)

${ }^{3}$ R. A. Marcus, J. Chem. Phys. 20, 359 (1952); R. A. Marcus and O. K. Rice, J. Phys. Colloid. Chem. 55, 894 (1951); R. A. Marcus, J. Chem. Phys. 43, 2658 (1965); 52, 1018 (1970).

${ }^{4}$ D. M. Wardlaw and R. A. Marcus, Chem. Phys. Lett. 110, 230 (1984).

${ }^{5}$ D. M. Wardlaw and R. A. Marcus, J. Chem. Phys. 83, 3462 (1985).

${ }^{6}$ D. M. Wardlaw and R. A. Marcus, J. Phys. Chem. 90, 5383 (1986).

${ }^{7}$ D. M. Wardlaw and R. A. Marcus, Adv. Chem. Phys. (in press).

${ }^{8}$ R. Viswanathan, L. M. Raff, and D. L. Thompson, J. Chem. Phys. 81, 3118 (1984).
${ }^{9}$ R. Viswanathan, L. M. Raff, and D. L. Thompson, J. Chem. Phys. 81,829 (1984).

10J. E. Adams, J. Chem. Phys. 78, 1275 (1983).

'S. C. Farantos, J. N. Murrell, and J. C. Hajduk, Chem. Phys. 68, 109 (1982).

'2L. B. Bhuiyan and W. L. Hase, J. Chem. Phys. 78, 5052 (1983).

${ }^{13}$ J. D. Doll, J. Chem. Phys. 74, 1074 (1981).

${ }^{14}$ J. D. Doll, Chem. Phys. Lett. 72, 139 (1980).

${ }^{15}$ A. J. Stace, J. Chem. Soc. Faraday Trans. 278,959 (1982).

${ }^{16}$ R. M. Stratt and J. E. Adams, J. Chem. Phys. 78, 2368 (1983).

${ }^{17}$ D. W. Noid, M. L. Koszykowski, M. Tabor, and R. A. Marcus, J. Chem. Phys. 72, 6169 (1980).

${ }^{18}$ S. W. Benson, Thermochemical Kinetics, 2nd ed. (Wiley, New York, 1976).

${ }^{19}$ D. M. Golden, J. Phys. Chem. 83, 108 (1979).

${ }^{20}$ E. V. Waage and B. S. Rabinovitch, Int. J. Chem. Kinet. 3, 105 (1971).

${ }^{21}$ D. B. Olson and W. C. Gardiner, Jr., J. Phys. Chem. 83, 922 (1979).

${ }^{22}$ A. W. Miziolek and G. C. Pimentel, J. Chem. Phys. 65, 4462 (1976).

${ }^{23}$ W. L. Hase, J. Chem. Phys. 57, 730 (1972); 64, 2442 (1976).

${ }^{24}$ M. Quack and J. Troe, Ber. Bunsenges. Phys. Chem. 78, 240 (1974).

${ }^{25}$ C. J. Cobos and J. Troe, Chem. Phys. Lett. 113, 419 (1985); J. Chem. Phys. 83, 1010 (1985); J. Phys. Chem. 90, 3485 (1986).

${ }^{26}$ J. F. LeBlanc and P. D. Pacey, J. Chem. Phys. 83, 4511 (1985).

${ }^{27}$ P. D. Pacey, J. Chem. Phys. 77, 3540 (1982).

${ }^{28}$ W. L. Hase, S. L. Mondro, R. J. Duchovic, and D. M. Hirst, J. Am. Chem. Soc. 109, 2916 (1987).

${ }^{29}$ S. Glasstone, K. J. Laidler, and H. Eyring, The Theory of Rate Processes (McGraw-Hill, New York, 1941).

${ }^{30}$ M. Quack and J. Troe, Ber. Bunsenges. Phys. Chem. 81, 329 (1977).

${ }^{31}$ D. G. Truhlar and B. C. Garrett, Annu. Rev. Phys. Chem. 35, 159 (1984).

${ }^{32}$ R. A. Marcus, J. Chem. Phys. 43, 2658 (1965).

${ }^{33}$ Wardlaw and Marcus have separated phase space into three regions and calculated the contribution from only those regions which are dynamically allowed. This is not done in the present canonical treatment because there is not a well defined energy to determine which configurations are allowed.

${ }^{34} \mathrm{H}$. Goldstein, Classical Mechanics, 2nd ed. (Addison-Wesley, Reading, 1980 ).

${ }^{35}$ In the present case this involves choosing points in configuration space by choosing $\theta_{i}$ randomly between 0 and $\pi$ and then choosing $\phi_{i}$, and $\psi_{i}$ randomly between 0 and $2 \pi$. The integral is then given by $\Sigma_{i \approx 1}^{N_{M C}} \exp \left(-\beta V_{i}\right)$ $\left(8 \pi^{2}\right)^{2} / N_{\mathrm{MC}}$.

${ }^{36}$ R. A. Kuharski and P. J. Rossky, J. Chem. Phys. 82, 5164 (1985).

${ }^{37}$ L. Schulman, Phys. Rev. 176, 1558 (1968).

${ }^{38}$ In the fixed-axis approximation the least classical action is approximated by the action for a rotation at constant angular velocity about the fixedaxis n. Kuharski and Rossky (Ref. 36) have shown that this action is the same as the least classical action to order $(\Delta t)^{2}$ and is thus valid for short times, which corresponds to large $P$ or high temperatures.

${ }^{39}$ D. A. McQuarrie, Statistical Mechanics (Harper and Row, New York, 1976).

${ }^{40}$ J. H. Van Vleck, Proc. Natl. Acad. Sci. U. S. A. 14, 178 (1928).

${ }^{41}$ D. J. Evans, Mol. Phys. 34, 317 (1977).

${ }^{42}$ D. J. Evans and S. Murad, Mol. Phys. 34, 327 (1977).

${ }^{43}$ J. D. Doll and L. E. Myers, J. Chem. Phys. 71, 2880 (1979); J. D. Doll, ibid. 81,3536 (1984).

${ }^{44}$ K. F. Stripp and J. G. Kirkwood, J. Chem. Phys. 19, 1131 (1951).

${ }^{45}$ J. P. Valleau and S. G. Whittington, in Statistical Mechanics, edited by B. J. Berne (Plenum, New York, 1977), p. 137.

${ }^{46}$ J. P. Valleau and G. M. Torrie, in Statistical Mechanics, edited by B. J. Berne (Plenum, New York, 1977), p. 169.

${ }^{47}$ For example, $\theta_{i}=\pi R_{1}, \phi_{i}=2 \pi R_{2}$, and $\psi_{i}=2 \pi R_{3}$, where $R_{1}, R_{2}$, and $R_{3}$ are random numbers between 0 and 1 .

${ }^{48}$ First, $\chi$ is set equal to $\cos (\Gamma / 2)$, where $\Gamma$ is the magnitude of the rotation. An axis of rotation is then chosen randomly by choosing a random point on a sphere of radius $\sqrt{1-\chi^{2}}$ and taking $\eta, \xi$, and $\xi$ as the three Cartesian coordinates of this point.

${ }^{49}$ The acceptance ratio is defined as the number of moves accepted divided by the number of moves attempted. It has been empirically determined that acceptance ratios of about $50 \%$ lead to the most rapid convergence. See, for example, J. A. Barker, Lattice Theories of the Liquid State (MacMillan, New York, 1963).

${ }^{50}$ Typical computational times on VAX 11/780 were, for the quantum calculations $30 \mathrm{~min}$, and for the classical calculations $6 \mathrm{~min}$. 
${ }^{51}$ The initial configuration for each of the quantum calculations was taken to be the configuration of minimum potential energy for the given $R^{+}$. At the start of the quantum calculations 100 initialization steps were performed before starting the evaluation of the integral. After this the integrands were summed up for $\mathbf{4 0} 000$ points. In the classical calculations no initialization procedure is needed and 50000 points were used in the calculation of the integral. The values of $\delta_{0}^{\text {fr }}$ used were $0.25,0.20,0.14,0.09$, for $T=300,500,1000$, and $2000 \mathrm{~K}$, respectively. The values of $\left(\delta_{0}^{\mathrm{Cl}}, \delta_{1}^{\mathrm{Cl}}\right)$ were $(1.0,0.22),(0.65,0.17),(0.45,0.09)$, and $(0.4,0.06)$ for the same four temperatures.

${ }^{52}$ E. Wigner, Phys. Rev. 40, 749 (1932).

${ }^{53}$ J. G. Kirkwood, Phys. Rev. 44, 31 (1933).

${ }^{54}$ H. Friedmann, Adv. Chem. Phys. 4, 262 (1976).

55J. G. Powles and G. Rickayzen, Mol. Phys. 38, 1875 (1979); 40, 1533 (1980). 